\title{
Renal replacement therapy in neonates with an inborn error of metabolism
}

Heeyeon Cho, MD, PhD

Department of Pediatrics, Samsung Medical Center, Sungkyunkwan University School of Medicine, Seoul, Korea

Hyperammonemia can be caused by several genetic inborn errors of metabolism including urea cycle defects, organic acidemias, fatty acid oxidation defects, and certain disorders of amino acid metabolism. High levels of ammonia are extremely neurotoxic, leading to astrocyte swelling, brain edema, coma, severe disability, and even death. Thus, emergency treatment for hyperammonemia must be initiated before a precise diagnosis is established. In neonates with hyperammonemia caused by an inborn error of metabolism, a few studies have suggested that peritoneal dialysis, intermittent hemodialysis, and continuous renal replacement therapy (RRT) are effective modalities for decreasing the plasma level of ammonia. In this review, we discuss the current literature related to the use of RRT for treating neonates with hyperammonemia caused by an inborn error of metabolism, including optimal prescriptions, prognosis, and outcomes. We also review the literature on new technologies and instrumentation for RRT in neonates

Key words: Continuous renal replacement therapy, Neonate, Inborn error of metabolism

\section{Introduction}

The etiology of neonatal hyperammonemia includes inborn errors of metabolism, transient hyperammonemia in the premature infant, severe perinatal asphyxia, total parenteral nutrition, and liver failure. ${ }^{1)}$ Inborn errors of metabolism causing hyperammonemia comprise several genetic disorders including urea cycle defects, organic acidemias, fatty acid oxidation defects, and certain disorders of amino acid metabolism. ${ }^{2)}$ The overall incidence of small molecule diseases causing congenital hyperammonemia is 1:9,160, among which the incidence of organic acidurias and urea cycle defects are 1:21,422 and 1:41,506, respectively. ${ }^{3)}$ The onset of hyperammonemia can occur during the neonatal period (40\%), infanthood (30\%), childhood (20\%), or adulthood (5\%-10\%).

Hyperammonemia is extremely neurotoxic, causing astrocyte swelling, brain edema, coma, severe disability, and even death. Thus, emergency treatment of hyperammonemia must be initiated before a precise diagnosis is established. ${ }^{4)}$ The aim of such medical treatment are to reduce precursor levels and catabolism while increasing anabolism. ${ }^{1)}$ Current practice guidelines for treating hyperammonemia include cessation of protein consumption, caloric intake $\geq 100 \mathrm{kcal} / \mathrm{kg}$, and use of medications such as insulin, arginine, carnitine, vitamins, benzoate/phenylbutyrate, and peroral carbamylglutamate. ${ }^{5,6)}$ In cases where the level of plasma ammonia does not respond to medical treatment, renal replacement therapy (RRT) such as hemodialysis (HD), peritoneal dialysis (PD), or continuous renal replacement therapy (CRRT) should be started before development of neurological impairments (Table 1). In neonates with hyperammonemia caused by an inborn error of metabolism, a few studies have suggested that PD, intermittent $\mathrm{HD}$, and CRRT are effective modalities to decrease the plasma
Corresponding author: Heeyeon Cho, MD, PhD Department of Pediatrics, Samsung Medical Center, Sungkyunkwan University School of Medicine, 81 Irwon-ro, Gangnam-gu, Seoul 06351, Korea Tel: +82-2-3410- 3535 Fax: +82-2-3410-0043 E-mail: choheeyeon@gmail.com https://orcid.org/0000-0003-3137-6054

Received: 2 October, 2018

Revised: 25 October, 2018

Accepted: 6 November, 2018
Copyright (C) 2019 by The Korean Pediatric Society

This is an open-access article distributed under the terms of the Creative Commons Attribution NonCommercial License (http://creativecommons.org/ licenses/by-nc/4.0/) which permits unrestricted noncommercial use, distribution, and reproduction in any medium, provided the original work is properly cited. 
Table 1. Current modalities of renal replacement therapy in neonates with hyperammonemia

\begin{tabular}{llll}
\hline Variable & \multicolumn{1}{c}{ Principle } & Efficiency of removal for small molecules & Tolerance \\
\hline Peritoneal dialysis & Diffusion+ultrafiltration & Poor & Good \\
Hemodialysis & Diffusion & Very high & Poor \\
Continuous venovenous hemofiltration & Ultrafiltration & Poor & Good \\
Continuous venovenous hemodiafiltration & Diffusion+Ultrafiltration & High & Good \\
\hline
\end{tabular}

level of ammonia. ${ }^{4,7-12)}$ However, there is little data on the optimal modality, prescription, or prognostic factors associated with RRT in neonates with hyperammonemia. In the following review, we discuss the current literature on the use of RRT for treating neonates with hyperammonemia caused by an inborn error of metabolism, including optimal prescriptions, prognosis, and outcomes. We also review the literature on new technologies for RRT in neonates.

\section{Use of RRT in neonates with hyperammonemia}

Prior to the 1990s, neonates with hyperammonemia were treated with PD because of the risk of hemodynamic instability and the technical challenges of performing extracorporeal dialysis. In 1989 there was a report of 4 neonates who presented with a coma secondary to hyperammonemia and were treated with PD within 16 and 120 hours, with clinical improvement observed in three patients within 16 to 72 hours of initiation of PD."

Ammonia in plasma diffuses easily through dialysis membranes; thus, removal of ammonia by extracorporeal dialysis is faster and more efficient compared to the use of PD for the removal of ammonia. Since the 1990s, there have been a few reports comparing PD, intermittent $\mathrm{HD}$, and CRRT for lowering the plasma level of ammonia in neonates with hyperammonemia. Arbeiter et al. ${ }^{8)}$ reported that the plasma ammonia level was significantly reduced by 50\% within $4.7 \pm 2.5$ hours with continuous venovenous $\mathrm{HD}$ whereas continuous PD achieved this result within $13.5 \pm 6.2$ hours, and plasma ammonia levels of $<200 \mu \mathrm{g} / \mathrm{dL}$ were achieved within $22.4 \pm 18.1$ hours using continuous venovenous $\mathrm{HD}$ whereas continuous $\mathrm{PD}$ achieved this result within 35.0 \pm 24 . 1 hours. This result suggests that CRRT using continuous venovenous HD can effectively and quickly reduce plasma ammonia. Lai et al. ${ }^{9}$ found that intermittent HD reduces plasma ammonia level by as much as 50\% after 1-2 hours compared with this result being achieved within 2-14.5 hours with continuous venovenous hemofiltration. In addition, they also reported that high-volume continuous venovenous hemofiltration results in good clearance of organic acids and ammonia in young children with inborn errors of metabolism and that CRRT in continuous venovenous hemofiltration can be considered as an alternative therapy if $\mathrm{HD}$ is not feasible due to technical challenges. ${ }^{9)}$ McBryde et al. ${ }^{10)}$ reported that initial therapy with HD is associated with improved survival and concluded that intermittent HD should be the first-line RRT modality for treatment of hyperammonemia, whereas CRRT should be used to prevent rebound after HD is stopped. Picca et al. ${ }^{11)}$ reported that plasma ammonia level decreases significantly within the first 24 hours irrespective of dialysis modality and that continuous venovenous HD achieves the highest ammonium clearance, suggesting that CRRT in continuous venovenous HD mode is the optimal modality for extracorporeal ammonium detoxification. In their study, the most relevant indicator of prognosis was coma duration before the start of dialysis. ${ }^{11)}$ In summary, although PD can be started immediately, it has a relatively slower rate of ammonia removal thus it can be difficult to balance ammonia formation. On the other hand, although $\mathrm{HD}$ is the most efficient way to remove ammonia, hypotension is a frequently cited complication, and ammonia level tends to rebound after HD is terminated. In CRRT, there is a limit to secure vascular access, as well as a lack of equipment appropriate for neonates. Nevertheless, high tolerance for CRRT is possible in neonates who are hemodynamically unstable, and the rebound effect of plasma ammonia is often not critical.

\section{Optimal prescription of CRRT in neonates with hyperammonemia}

Spinale et al. ${ }^{13)}$ reported cases of 2 neonates with hyperammonemia who were diagnosed with ornithine transcarbamylase deficiency and received high-dose CRRT. Using dialysis/replacement flow rates of $8,000 \mathrm{~mL} / \mathrm{hr} / 1.73 \mathrm{~m}^{2}$, which is 4 fold higher than the usual rate used for acute kidney injury, they decreased the level plasma ammonia to $<400 \mu \mathrm{mol} / \mathrm{L}$ and $<100 \mu \mathrm{mol} / \mathrm{L}$ within 3 and 10 hours, respectively, of initiating CRRT. ${ }^{13)}$

Likewise, Hanudel et al. ${ }^{14)}$ reported several cases of inborn errors of metabolism treated with a biphasic, high-dose CRRT strategy consisting first of a dialysis flow rate of 5,000 mL/hr (40,000 mL/ $\mathrm{hr} / 1.73 \mathrm{~m}^{2}$ ) to rapidly decrease plasma ammonia level, followed by a second step with a dialysis flow rate of $500 \mathrm{~mL} / \mathrm{hr}(4,000 \mathrm{~mL} / \mathrm{hr} / 1.73$ $\mathrm{m}^{2}$ ) to prevent a rebound of plasma ammonia. ${ }^{14)}$ Using this biphasic dialytic treatment strategy, a rapid reduction in ammonia was achieved without rebound during a single dialysis session. Kim et al. ${ }^{15)}$ reported that the median time to reduce initial ammonia level by at least 50\% is 12.8 hours with a median ultrafiltration rate (UFR) at the initiation of CRRT of 2,288.4 mL/hr/ $1.73 \mathrm{~m}^{2}$, with a survival rate during hospitalization of 83.3\%. The study by Kim et al. ${ }^{15)}$ suggested that it is necessary to determine UFR according to initial plasma ammonia level. The mode of continuous venovenous $\mathrm{HD}$ with high 
dialysate flow is supposed to be the best available option. However, there is little data regarding the optimal prescription for CRRT to improve survival in neonates with an inborn error of metabolism, and further research is necessary.

\section{Prognosis and outcomes in neonates with hyper- ammonemia}

There are a few known prognostic factors in neonates with hyperammonemia receiving RRT. First, duration of coma before starting RRT has been reported as a significant prognostic factor. Specifically, Picca et al. reported that coma duration $<33$ hours is prognostic of a good outcome. ${ }^{11)}$ Pela et al. ${ }^{16)}$ also reported that outcomes are related to coma duration caused by hyperammonemia and that PD may be effective for neonatal hyperammonemia because it can be administered quickly. Taken together, these findings support the importance of prompt medical management with RRT, which may influence patient outcomes. Enns et al. ${ }^{17)}$ reported that good outcomes are associated with prompt recognition of inborn errors of metabolism and rapid initiation of medical treatment with both sodium phenylacetate and sodium benzoate, in conjunction with other therapies such as intravenous arginine hydrochloride. Enns et al. ${ }^{17)}$ suggested that HD may be needed to control hyperammonemia, especially in pediatric patients that do not respond to medical management.

RRT modality and the rate of plasma ammonia removal may also be associated with patient outcomes. Schaefer et al. ${ }^{18}$ reported that, among eight children presenting with hyperammonemic coma, four with the most rapid dialytic ammonia removal rate (50\% reduction within 7 hours) survived, whereas slower toxin removal was always associated with a lethal outcome. McBryde et al. ${ }^{10)}$ reported that initial therapy with $\mathrm{HD}$ was associated with improved survival, while delayed time to RRT ( $>24$ hours) was associated with increased mortality. Arbeiter et al. ${ }^{8)}$ reported that $18 \%$ of their continuous venovenous HD patients died compared with 50\% of those undergoing continuous PD, suggesting that patient outcomes may be associated with RRT modality. However, a more recent study indicated that in patients undergoing extracorporeal dialysis, the duration of coma before dialysis is longer than in those with PD and that there is no difference in short-term outcome between PD and extracorporeal dialysis. ${ }^{19)}$ The same study also suggested that total coma duration and plasma ammonia level before RRT are risk factors for death. ${ }^{19)}$

Lastly, patient conditions such as presenting vital signs may be associated with outcomes. Westrope et al. ${ }^{20)}$ reported that precontinuous venovenous hemofiltration conditions of neonates such as the PRISM (Pediatric Risk of Mortality) score and requirement for cardio-active medication are prognostic of patient outcomes. In summary, recent reports suggest that the most relevant indicator for prognosis is duration of coma prior to the start of dialysis. In addition, there is ongoing controversy as to whether the efficacy of initial dialysis has an effect on outcomes, because the efficacy of dialysis is often evaluated according to changes in plasma ammonia level, which is affected by other factors as well. As a result, it can be difficult to differentiate the influence of ammonia dialysis clearance from the effects of other variables.

\section{Obstacles to RRT and introduction of new equipment for neonates}

A retrospective study reported that infants undergoing CRRT have acceptable outcomes overall and that the outcomes of infants weighing 3-10 kg are similar to those of older patients. ${ }^{211}$ Infants with metabolic disorders have good outcomes, while neonates weighing less than $3 \mathrm{~kg}$ and with certain diagnoses such as congenital anomalies have worse outcomes. ${ }^{20)}$ There have been a few reports on outcomes in Korean neonates treated with CRRT. ${ }^{22,23)}$ Lee and $\mathrm{Cho}^{22)}$ analyzed the factors associated with outcomes in 34 neonates undergoing CRRT and reported an overall mortality of 50\% and that neonates with a higher percentage of fluid overload and a higher level of serum creatinine at the initiation of CRRT had worse outcomes.

The obstacles of CRRT in neonates include a large circuit volume, difficulty in fluid balance, and difficulty in establishing vascular access lines. The relatively large circuit volume $(\geq 60 \mathrm{~mL})$ needed for blood priming carries several risks. A detailed fluid balance is not possible, and the current instruments used for CRRT are not licensed for babies weighing less than $8 \mathrm{~kg}$. Likewise, 7F dual-lumen vascular access lines and blood flow rates of $40 \mathrm{~mL} / \mathrm{min}$ are usually recommended for efficient CRRT; however, this can be difficult to establish in neonates.

A new device for CRRT in patients $<10 \mathrm{~kg}$ was recently introduced, namely, the Cardio-Renal Pediatric Dialysis Emergency Machine (CARPEDIEM). The CARPEDIEM is the result of a 5-year project aimed at creating a new miniaturized CRRT machine for neonates and small infants $(2.5 \mathrm{~kg}){ }^{24-26)}$ The CARPEDIEM utilizes reduced priming volumes ( $27 \mathrm{~mL}$ including filter) and has the capacity to accurately handle very low blood and ultrafiltration flow rates (roller pump at a flow rate as low as 5-50 $\mathrm{mi} / \mathrm{min}$ with the ultrafiltration and replacement fluid pumps operating at 0-10 $\mathrm{mL} / \mathrm{min}){ }^{24)}$ The filters of the CARPEDIEM have different surface areas $\left(0.075,0.15\right.$, and $\left.0.25 \mathrm{~m}^{2}\right)$ that are appropriate for very small babies. ${ }^{24)}$ The first-in-human use of the CARPEDIEM was in 2013, where a neonatal patient received CRRT with CARPEDIEM (heparin anticoagulation). ${ }^{24)}$

The CARPEDIEM was originally designed for continuous venovenous hemofiltration in neonatal and pediatric patients and is limited in that adequate convective clearance may not be achieved 


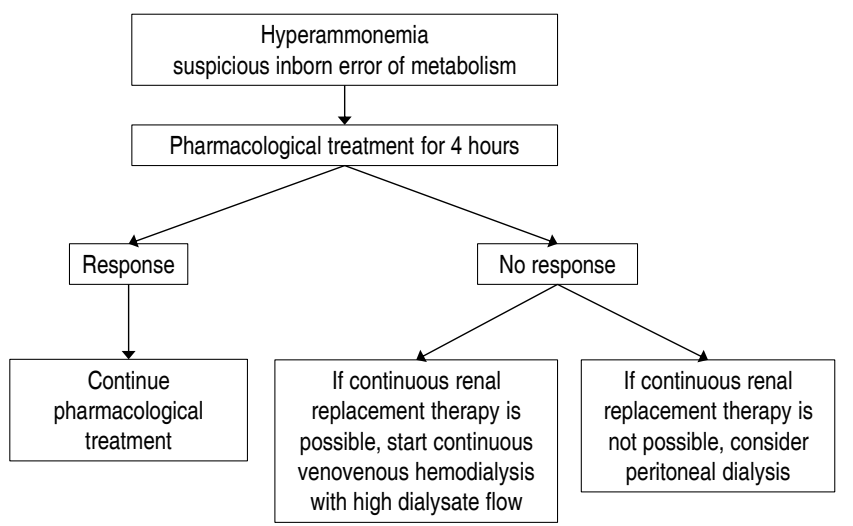

Fig. 1. The approach of renal replacement therapy for neonates with hyperammonemia.

because of limited blood flow. ${ }^{25)}$ Thus, there is an ongoing need for other medical technologies (catheters, fluids, and monitors) designed for small pediatric patients. Indeed, other dialysis machines for small babies have been developed, including the Newcastle infant dialysis and ultrafiltration system (Nidus) ${ }^{27)}$ developed in 1995, which consists of a novel syringe-driven $\mathrm{HD}$ circuit with a circuit volume of $13 \mathrm{~mL}$ that is designed for 24 hours continuous use. In 2005 , Nidus was used to treat 4 babies (800 g-3.4 kg) with a singlelumen access line and no blood-priming. ${ }^{27)}$ In 2014, Nidus was used to repeatedly withdraw $5-12.5 \mathrm{~mL}$ aliquots of blood from a single-lumen central venous line, pass and return the blood across a dialysis filter, and then send it back to the baby. ${ }^{27)}$ The prescriptions for Nidus include a blood flow rate of $20 \mathrm{~mL} / \mathrm{min}$, UFR of 0-60 mL/ $\mathrm{hr}$, and heparin anticoagulation. ${ }^{27)}$ Beyond CARPEDIEM and Nidus, there are relatively few reports of other RRT machines for small babies. ${ }^{28)}$ Further research on the optimal mode and prescription for RRT in small babies is needed, along with the development of new equipment.

\section{Conclusions}

RRT can be used to achieve a rapid decrease in plasma ammonia level, and continuous venovenous HD with a high dialysate flow rate appears to be the best available option. Severe hyperammonemia can be reversed by pharmacological treatment alone in 1/3 of patients, and 4 hours appears to be a reasonable time to allow for pharmacological treatment to take effect before initiating RRT (Fig.1). Although newly developed machines for small pediatric patients have been introduced, there is an ongoing need for research into optimal modalities, dosing according to primary disease (inborn errors of metabolism vs. acute kidney injury), and optimal anticoagulant therapy in neonates undergoing CRRT.

\section{Conflict of interest}

No potential conflict of interest relevant to this article was reported.

\section{References}

1. Auron A, Brophy PD. Hyperammonemia in review: pathophysiology, diagnosis, and treatment. Pediatr Nephrol 2012;27:207-22.

2. Lanpher B, Brunetti-Pierri N, Lee B. Inborn errors of metabolism: the flux from Mendelian to complex diseases. Nat Rev Genet 2006;7:44960.

3. Dionisi-Vici C, Rizzo C, Burlina AB, Caruso U, Sabetta G, Uziel G, et al. Inborn errors of metabolism in the Italian pediatric population: a national retrospective survey. J Pediatr 2002;140:321-7.

4. McBryde KD, Kudelka TL, Kershaw DB, Brophy PD, Gardner JJ, Smoyer WE. Clearance of amino acids by hemodialysis in argininosuccinate synthetase deficiency. J Pediatr 2004;144:536-40.

5. Summar M. Current strategies for the management of neonatal urea cycle disorders. J Pediatr 2001;138(1 Suppl):S30-9.

6. Häberle J, Boddaert N, Burlina A, Chakrapani A, Dixon M, Huemer M, et al. Suggested guidelines for the diagnosis and management of urea cycle disorders. Orphanet J Rare Dis 2012;7:32.

7. Gortner L, Leupold D, Pohlandt F, Bartmann P. Peritoneal dialysis in the treatment of metabolic crises caused by inherited disorders of organic and amino acid metabolism. Acta Paediatr Scand 1989;78: 706-11.

8. Arbeiter AK, Kranz B, Wingen AM, Bonzel KE, Dohna-Schwake C, Hanssler L, et al. Continuous venovenous haemodialysis (CVVHD) and continuous peritoneal dialysis (CPD) in the acute management of 21 children with inborn errors of metabolism. Nephrol Dial Transplant 2010;25:1257-65.

9. Lai YC, Huang HP, Tsai IJ, Tsau YK. High-volume continuous venovenous hemofiltration as an effective therapy for acute management of inborn errors of metabolism in young children. Blood Purif 2007;25: 303-8.

10. McBryde KD, Kershaw DB, Bunchman TE, Maxvold NJ, Mottes TA, Kudelka TL, et al. Renal replacement therapy in the treatment of confirmed or suspected inborn errors of metabolism. J Pediatr 2006; 148:770-8.

11. Picca S, Dionisi-Vici C, Abeni D, Pastore A, Rizzo C, Orzalesi M, et al. Extracorporeal dialysis in neonatal hyperammonemia: modalities and prognostic indicators. Pediatr Nephrol 2001;16:862-7.

12. Kim HJ, Park SJ, Park KI, Lee JS, Eun HS, Kim JH, et al. Acute treatment of hyperammonemia by continuous renal replacement therapy in a newborn patient with ornithine transcarbamylase deficiency. Korean J Pediatr 2011;54:425-8.

13. Spinale JM, Laskin BL, Sondheimer N, Swartz SJ, Goldstein SL. Highdose continuous renal replacement therapy for neonatal hyperammonemia. Pediatr Nephrol 2013;28:983-6.

14. Hanudel M, Avasare S, Tsai E, Yadin O, Zaritsky J. A biphasic dialytic strategy for the treatment of neonatal hyperammonemia. Pediatr Nephrol 2014;29:315-20.

15. Kim JY, Lee Y, Cho H. Optimal prescriptions of continuous renal replacement therapy in neonates with hyperammonemia. Blood Purif 2018;14:1-7.

16. Pela I, Seracini D, Donati MA, Lavoratti G, Pasquini E, Materassi M. Peritoneal dialysis in neonates with inborn errors of metabolism: is it really out of date? Pediatr Nephrol 2008;23:163-8. 
17. Enns GM, Berry SA, Berry GT, Rhead WJ, Brusilow SW, Hamosh A. Survival after treatment with phenylacetate and benzoate for ureacycle disorders. N Engl J Med 2007;356:2282-92.

18. Schaefer F, Straube E, Oh J, Mehls O, Mayatepek E. Dialysis in neonates with inborn errors of metabolism. Nephrol Dial Transplant 1999;14:910-8.

19. Picca S, Dionisi-Vici C, Bartuli A, De Palo T, Papadia F, Montini G, et al. Short-term survival of hyperammonemic neonates treated with dialysis. Pediatr Nephrol 2015;30:839-47.

20. Westrope C, Morris K, Burford D, Morrison G. Continuous hemofiltration in the control of neonatal hyperammonemia: a 10-year experince. Pediatr Nephrol 2010;25:1725-30.

21. Symons JM, Brophy PD, Gregory MJ, McAfee N, Somers MJ, Bunchman TE, et al. Continuous renal replacement therapy in children up to 10 kg. Am J Kidney Dis 2003;41:984-9.

22. Lee ST, Cho H. Fluid overload and outcomes in neonates receiving continuous renal replacement therapy. Pediatr Nephrol 2016;31: 2145-52.

23. Sohn YB, Paik KH, Cho HY, Kim SJ, Park SW, Kim ES, et al. Continuous renal replacement therapy in neonates weighing less than 3 kg. Korean J Pediatr 2012;55:286-92.

24. Ronco C, Garzotto F, Brendolan A, Zanella M, Bellettato M, Vedovato $\mathrm{S}$, et al. Continuous renal replacement therapy in neonates and small infants: development and first-in-human use of a miniaturised machine (CARPEDIEM). Lancet 2014;383:1807-13.

25. Lorenzin A, Garzotto F, Alghisi A, Neri M, Galeano D, Aresu S, et al. CVVHD treatment with CARPEDIEM: small solute clearance at different blood and dialysate flows with three different surface area filter configurations. Pediatr Nephrol 2016;31:1659-65.

26. Vidal E, Garzotto F, Parolin M, Manenti C, Zanin A, Bellettato M, et al. Therapeutic plasma exchange in neonates and infants: successful use of a miniaturized machine. Blood Purif 2017;44:100-5.

27. Coulthard MG, Crosier J, Griffiths C, Smith J, Drinnan M, Whitaker M, et al. Haemodialysing babies weighing $<8 \mathrm{~kg}$ with the Newcastle infant dialysis and ultrafiltration system (Nidus): comparison with peritoneal and conventional haemodialysis. Pediatr Nephrol 2014;29: 1873-81.

28. Askenazi D, Ingram D, White S, Cramer M, Borasino S, Coghill C, et al. Smaller circuits for smaller patients: improving renal support therapy with Aquadex ${ }^{\mathrm{TM}}$. Pediatr Nephrol 2016;31:853-60. 